\title{
Nuclear Research with the Electromagnetic Probe
}

\author{
A Progress Report for the renewal of \\ Contract DE-FG03-88ER40439
}

\section{Stanford University}

\section{DISCLAIMER}

This report was prepared as an account of work sponsored by an agency of the United States Government. Neither the United States Government nor any agency thereof, nor any of their employees, makes any warranty, express or implied, or assumes any legal liability or responsibility for the accuracy, completeness, or usefulness of any information, apparatus, product, or process disclosed, or represents that its use would not infringe privately owned rights. Reference herein to any specific commercial product, process, or service by trade name, trademark, manufacturer, or otherwise does not necessarily constitute or imply its endorsement, recommendation, or favoring by the United States Government or any agency thereof. The views and opinions of authors expressed herein do not necessarily state or reflect those of the United States Government or any agency thereof.

Stanford, CA 94305

January 1990

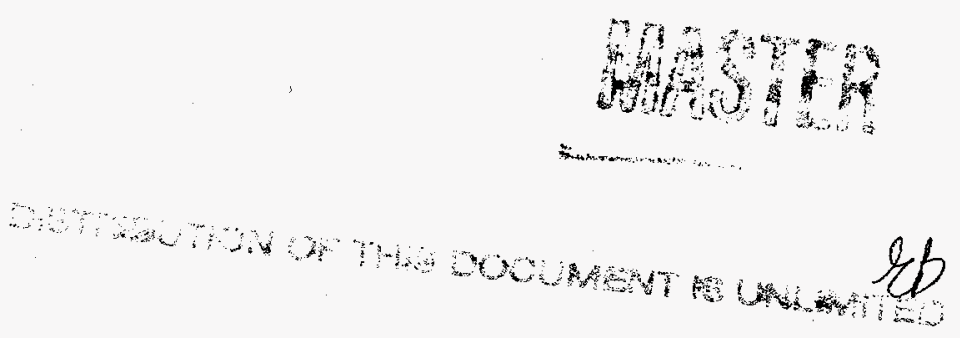




\section{DISCLAIMER}

Portions of this document may be illegible in electronic image products. Images are produced from the best available original document. 


\section{Table of Content}

\section{Page}

I. Introduction

3

II. Progress report 4

II.1 Experiments at SLAC 4

$\begin{array}{ll}\text { II.2 Experiments at SACLAY } & 6\end{array}$

III. Contribution to Futur Projects 6

IV. Plan for research in $90-91 \quad 7$

V. Budget 8

V.1 Capital Equipment $\quad 8$

V.2 Travel 9

$\begin{array}{ll}\text { VI. Publications } & 10\end{array}$ 


\section{Introduction}

The eletromagnetic probe is used to address some of the forefront questions in nuclear physics. Two questions are of special interest in this project, one is related to the electromagnetic properties of the nucleon in the nuclear medium, the other concerns the transition between nucleons-mesons and quarks-gluons degrees of freedom when describing nuclei at medium energies.

The electromagnetic properties of free protons have been extensively studied and are used as basic input to describe any of the electric or magnetic properties of nuclei. However, inclusive and semi-exclusive experiments measurements in the quasielastic and the deep inelastic region seem to indicate that the properties of bound nucleons are modified significantly in the nuclear medium. It is therefore of first importance to understand how the free properties of nucleons are modified in order to have a realistic description of nuclei. It was suggested, for example, that nucleons are swollen in nuclei. The physical consequences of such an idea are of great impact on the description of nuclei.

The nature of degrees of freedom needed to describe nuclei in the most economical and efficient maner is of special interest to the nuclear physics community. It is clear that the ultimate degrees of freedom relevant for the strong interactions are quarks and gluons and QCD is the theory that starts from these degrees of to explain nuclei. The test of QCD has been performed in many experiments in the perturbative regime where the running coupling constant is small and wehere physical processes are therefore calculable. In fact, it is widely accepted that perturbative QCD describe properly the physical quantities of interest. From the theoretical stand point it is very difficult to perform QCD calculations for the non perturbative regime. Unfortunatly for most of nuclear physics those calculations have to be carried in the low energy regime. Up to now, nucleons and mesons degrees of freedom have been used to describe most of our knowledge in nuclear physics with remarkable succes. However there is a growing interest in studying the transition region where one hope to see the sign of quark degrees of freedom emerging. It is the study of QCD in nuclei from low to high energies that is of interest for the nuclear physics community. Some of the experiments proposed in this project explore a small window in this study. 
We have performed two experiments at SLAC using the Nuclear Physics Injector. One will allow the separation of the transverse and longitudinal response functions of ${ }^{3} \mathrm{He},{ }^{4} \mathrm{He},{ }^{27} \mathrm{Al}$ and ${ }^{56} \mathrm{Fe}$ to electron scattering at $Q^{2}=1(\mathrm{GeV} / \mathrm{c})^{2}$. The other measured the photodisintegration of deuterium at large photon energies (.8 to $1.6 \mathrm{GeV}$ ), looking at deviation from the standard calculations including nucleons, mesons exchange currents and resonance components. The sensitivity of the reaction mechanism to quark degrees of freedom is also tested.

We have also extended to a larger kinematical range two experiments performed at the Saclay linear Accelerator center. On one hand the study of the nucleon momentum distribution of ${ }^{4} \mathrm{He}\left(e, e^{\prime}\right)$ is to be extracted from both the transverse and longitudinal exclusive response functions in the quasielastic region. A comparison of these two response functions as a function of $Q^{2}$ up to $.8(\mathrm{GeV} / \mathrm{c})^{2}$ will provide information on the density effect of the modification of the free nucleon properties in nuclear medium. On the other hand the separation of the transverse and longitudinal separation of the exclusive response functions in the region of the three-body break up of ${ }^{3} \mathrm{He}$ is performed aiming to study the proton-proton correlation in a direct way through ${ }^{3} \mathrm{He}\left(e, e^{\prime} p\right) p n$ reaction. A serious progress has been achieved in performing "complete" theoretical calculations on ${ }^{3} \mathrm{He}$ that would allow to understand the proton-proton correlation in ${ }^{3} \mathrm{He}$.

\section{Progress Report}

\section{II.1. Experiments at SLAC.}

\section{II.1.a High $Q^{2}$ Transverse Longitudinal Quasielastic Scattering.}

The analysis of the experiment known as NE-9 at the nuclear physics program at SLAC is almost completed. Preliminary results exist at this stage, and a thorough comparison between the analysis carried at the University of Virginia and at that of Stanford University is performed determining the final results. The main difficulties in this experiment are related to the determination of the absolute cross sections measured at backward and small scattered electron energies. The background from unknown origins has been the main concern in our careful study. The third pass of the analysis is underway and should be over before spring.

Figure 1. shows some of the preliminary results. Inclusive cross sections on 
${ }^{56} \mathrm{Fe}$ at forward and backward angles. Radiative corrections will be applied and transverse and longitudinal response functions are to be extracted from those cross sections. These data were presented at the Asilomar meeting of the American Physical Society meeting at Asilomar, October 11, 1989. and through an invited talk at The Electromagnetic Probe Workshop at the date and location.

We are invited to present the results of ${ }^{56} \mathrm{Fe}$ at the American Physical Society at the Washington Meeting in a plenary session. We are also invited to present the results on all nuclei at the Gordon Conference in the month of August of this year.

\section{II.1.b Two body Photodisintegration of deuterium between .6 and 1.8 GeV}

After a successful run of the experiment known as NE-8 and data analysis interesting results due to this experiment are debated in the nuclear physics community. The early experimental data on the differential cross section of photodisintegration at $\theta_{c m}=90^{\circ}$ up to $800 \mathrm{MeV}$ photon energy are well described by a traditional calculations including meson exchange currents, the $\Delta$ resonance contribution and final state interactions. However the new measured data up to $1.6 \mathrm{GeV}$ photon energies disagree significantly with those same calculations. In the energy range between 0.8 and $1.8 \mathrm{GeV}$ the data appear to obey a simple scaling law predicted by the constituent-rules assuming parton degrees of freedom for the deuterium and nucleons. The question of what degrees of freedom are relevant to describe nuclei, in an economical way and with predicting power, is far to be settled between the two schools of thoughts. One school deals with nucleon and meson degrees of freedom and the other school uses quarks as the basic constituents to describe all phenomena in nuclear physics. Deuterium is the simplest nuclear system one can imagine and it is of paramount importance to interpret the data properly since the physics consequences will change our view of the description approach one might have of the nuclear system in the intermediate energy regime. There are more experiments proposed on this simple nucleus to study those issues. This work has been published in the following reference; J. Napolitano et al. Phys. Rev. Lett. 61(1988)2530. A regular paper is under preparation for submission to Phys. Rev. C. A new proposal to extend this experiment to higher energies and using the 8 
$\mathrm{GeV}$ spectrometer with a modified detector package to identify protons has been submitted to the NPAS program at SLAC.

\section{II.2 Experiments at Saclay.}

11.2.a Transverse and Longitudinal Exclusive Response Functions of ${ }^{3} \mathrm{He}$ and ${ }^{4} \mathrm{He}$

The extension to higher momentum transfers of the transverse and longitudinal exclusive response functions of the reaction ${ }^{4} \mathrm{He}\left(e, e^{\prime} p\right)^{3} \mathrm{H}$ in the quasielastic region was carried out in June 1990 during three weeks. The longitudinal over transverse ratio, performed on the two-body break-up part of the response, allows to study the electromagnetic current of the proton in ${ }^{4} \mathrm{He}$. In other words the aim of the experiment is not to concentrate on the nuclear wave function of the nucleus but rather extract the electromagnetic properties of the proton in the nucleus. The ${ }^{4} \mathrm{He}$ nucleus was chosen because of the reasonably sophisticated calculations that exist to describe it. It has been found, after all known nuclear effect corrections, that the ratio of the electric to magnetic form factor of the proton in the nucleus is different by almost $20 \%$ when compared to the free proton ratio. These data do not comfirm or rule out a momentum transfer dependence of this ratio leaving open the question of how much swollen the nucleon is in the nucleus. However it confirms for a light and calculable nucleus the quenching observed in heavier nuclei. It is worth noticing that a similar effect was observed indirectly on heavy nuclei through a sum rule analysis of inclusive experiments, however, in that case the theoretical models to describe those nuclei are, in the contrary of ${ }^{4} \mathrm{He}$, less reliable. It is also important to point out that protons in ${ }^{3} \mathrm{He}$ do not display such effect. Experiments are underway to improve the precision of the data in order to have a better assessment of the momentum transfer dependence. More theoretical calculations are underway to interpret the observed ratio. The first results of the experiment have been published in the following reference A. Magnon et al., Phys. Lett. B222 (1989)352

The experiment to study proton-proton correlations in nuclei was performed at the same time in june 1990 . We hope to separate the contribution of $\mathrm{pp}$ pairs from that of np pairs in the exclusive response function of the three-body breakup of ${ }^{3} \mathrm{He}$ by separating of the transverse and longitudinal parts of the response 
function. The analysis of this experiment is underway and the results are expected this year. This experiment will be crucial as a first step towards understanding the nucleon-nucleon interaction in nuclei. A proposal of an extension of this experiment is being prepared for the Bates linear acceleractor.

\section{Contributions to Future Projects (CEBAF)}

We are are part of the Hall A collaboration at CEBAF, taking the responsibility of building the electron beam polarimeter for the polarized beam sent to Hall $A$. Two techniques of polarimetry are possible they are called Compton or Moller polarimetry referring to the scattering used (real photons or electrons) in measuring the polarization of the electron beam. We have been looking at these two techniques and found them very complementary and both are to be used at CEBAF because of the dynamical range of the current and energies of the CEBAF beam. We have been contributing to the design report of Hall A describing the beam line. Our committment to CEBAF is strong since we beleive that this facility will play a crucial role in understanding nuclear systems using the electromagnetic probe.

As part of the HERMES collaboration that will measure the spin structure functions of the nucleon at HERA we have been studying the design of a longitudinal beam polarimeter for the experiment. Our goal is to bring our experience in bulding this polarimeter to develop the beam polarimetry at CEBAF. I should point out that the measurement of polarization at CEBAF is more dificult because of the range of energies of backscattered photons we will be detecting.

\section{Plan for research in $90-91$}

Our first priority in 1990 is to complete the analysis of NE-9 and publish most of the important results on all nuclei. We expect to have two letters before the end of this year. We will be preparing a long paper later to describe the full experiment with its physics conequences.

Beam time has been approved for this next spring to extend the ${ }^{3} \mathrm{He}\left(e, e^{\prime} \mathrm{p}\right)^{3} \mathrm{H}$ experiment at Saclay. The transverse and longitudinal separation of the exclusive response functions in the three body break up region will be extended to higher momentum transfers. The longitudinal response will be directly sensitive to the proton-proton correlation at these transfers. 
A proposal for measuring Double Charge Pion Exchange on the Calcium isotopes has been presented to the PAC at Los Alamos and beam time will be available this next summer to perform the experiment.

We will start designing the electron beam polarimeter for the Hall A at CEBAF. More work will be directed in defining the physics and detectors of $\mathrm{Hall} \mathrm{A}$ in order to have the best program of physics and the right tools to perform it. With the agreement of the CEBAF management we will put a proposal to the Department of Energy for Research and Development to build the polarimeter this next summer.

The final version of the proposal for the measurement of the spin structure functions of the proton and the neutron has been submitted to the HERA managment. Stanford contribution will be the measurement of the longitudinal polarization of the beam after the spin rotators in the west Hall at HERA. More work is needed to define the detectors for this measurement. After the next PAC at HERA this spring, we will know how to procede with respect to putting the proposal through DOE for review.

\section{Budget 90-91}

The proposed budget for this year is covering two months summer salary of the principal investigator, a year salary of a graduate student and $15 \%$ contribution to the salary of a secretary. We are also asking funds to cover part of the expenses for a collaboration visit of Dr. J. M. Laget from Saclay for one week this next summer.

We will be working on the developement of the electron beam polarimeter at CEBAF and machine shop labor will be needed. The capital equipment and the travel part of the budget are described separately.

\section{V.1 Capital equipment description}

The two items listed in the capital equipment are respectively a time digital converter electronic module and a disk subsystem for data storage. As it will be explicitely stated in our next proposal to DOE, we are providing part of the technical support for building the electron beam polarimeter at CEBAF. This is a very crucial instrument of the Hall $A$ beam line for all experiments that require the polarized electron beam. The Electronic module we are asking for will be used 
for research and developement that we are undertaking for building the electron beam polarimeter. The disk subsystem is used as a dedicated media for running simulations on the polarimeter. We are studying all possible backgrounds in the interaction region between the laser beam and the electron beam. The program used for these simulations is GEANT which is very large and takes a significant amount of disk space. The subsystem we are asking for will allow us to run and store the information in a efficient manner.

Lecroy 2228A Octal 11 bit TDC w/ NIM inputs

Eakins EAE21 Disk subsystem

$\$ 1294$

Total

$\$ 3249$ 


\section{V.1 Travel}

The travel budget in the U.S. will cover expenses for the collaboration meetings at CEBAF Newport News and Charlottesville Virginia and performing an experiment at Los Alamos

The foreign travel budget include expenses for Saclay to perform the continuation of ${ }^{3} \mathrm{He}\left(e, e^{\prime} p\right)$ experiment and to HERA in Germany for the collaboration meetings to measure the spin structure functions of the proton and neutron.

Domestic travel

five trips to Virginia

$\$ 3500$

one trip to Los Alamos

$\$ 500$

Total

$\$ 4000$

Foreign travel

Two trips to Saclay, France

$\$ 3200$

One trip to Hamburg, Germany

$\$ 1500$

Total

$\$ 4700$ 


\section{Publications}

\section{V.1 Articles}

A. Magnon, M. Bernheim, M. Brussel, G. P. Capitani, E. De Sanctis, S. Frullani, F. Garibaldi, A. Gerard, H. Jackson, J. M. Legoff, C. Marchand, Z.E. Meziani, J. Morgenstern, J. Picard, D. Reffay, S. Turck-Chieze, P. Vernin, A. Zghiche, "A Study of the Electrodisintegration Reaction ${ }^{4} \mathrm{He}\left(e, e^{\prime} p\right)^{3} \mathrm{H}$ with TransverseLongitudinal Separation" Phys. Lett. B222 (1089)352

D.B. Day, J.S. McCarthy, Z.E. Meziani, R.Minehart, R. Sealock, S.T. Thornton, J. Jourdan, I. Sick, B.W. Filippone, R.D. McKeown, R.G. Milner, D. H. Potterveld, and Z. Szalata, "Nuclear Matter Response Function", Phys. Rev. C 40(1989)1011

B.W. Filippone, R.D.McKeown, R.G. Milner, D. H. Potterveld, D.B. Day, J.S. McCarthy, Z.E. Meziani, R.Minehart, R. Sealock, S.T. Thornton, J. Jourdan, I. Sick, and Z. Szalata, "Nuclear Response Function for $x>1$ ", submitted to Phys. Rev. C (1989).

M. Bernheim, M. Brussel, G. P. Capitani, E. De Sanctis, S. Frullani, F. Garibaldi, J. M. Le Goff, H. Jackson, A. Magnon, C. Marchand, Z.E. Meziani, J. Morgenstern, J. Picard, D. Reffay-Pikeroen, S. Turck-Chieze, P. Vernin, A. Zghiche, "QuasiElastic Electron Scattering in Nuclei" Topical Workshop on Two-Nucleon Emission Reactions, Elba International Physics Center, Italy September 19-23,1989 to be published in the proceedings by World Scientific Publishing Co.

\section{V.2 Invited talks}

Z. Meziani, "Study of Nucleon-Nucleon Correlations and Final State Interactions using the Electromagnetic Probe.", Second LAMPF International Workshop on Pion Double Charge Exchange, August 9-11, 1989 to be published in the proceedings by World Scientific Publishing Co.

Z.E. Meziani, "High $Q^{2}$ Quasielastic Longitudinal/Transverse Separation" ,High Energy Electro-Nuclear Physics Workshop, Meeting of the Division of Nuclear Physics Pacific Grove CA, October 10-13, 1989, Bull. Am. Phys. Soc. 


\section{V.3 Abstracts}

J.P. Chen, S. Auffret, L. Chinitz, D. Day, K. Giovanetti, J.S. McCarthy, R.C. Minehart, O. Rondon-Aramayo, R.M. Sealock, S.T. Thornton, Z.E. Meziani, L.C. Dennis, K.W. Kemper, I. Sick, B.W. Fillipone, J. Jourdan, R.D. McKeown, R.G. Milner, D. Potterveld, R. Walker, C. Woodward, J. Morgenstern, and J. Mougey "Measurements of the Transverse and Longitudinal Response Functions for Several Nuclei at $Q^{2}$ Near $1(\mathrm{GeV} / \mathrm{c})^{2 n}$, Bull. Am. Phys. Soc. $\underline{33}, 1593$ (1988).

A. Magnon, M. Bernheim, M. Brussel, G.P. Capitani, E. De Sanctis, S. Frullani, F. Garibaldi, A. Gerard, H. Jackson, J.M. Legoff, C. Marchand, Z.E. Meziani, J. Morgenstern, J. Picard, D. Reffay, S. Turck-Chieze, P. Vernin, A. Zghiche, "Exclusive experiments on Few Nucleon systems at SACLAY", Contribution to the 4th Workshop on perspectives in Nuclear Physics at Intermediate energies, Trieste, Italy, May 8-12, 1989.

S. Freedman, D. Geesaman, R. Gillman, M. Green, R.J. Holt, H.E. Jackson, E.R. Kinney, R. Kowalczyk, C. Marchand, J. Napolitano, J. Nelson, D. Potterveld, B,. Zeidman, R. Segel, T.-Y. Tung, D. Beck, G. Boyd, D. Collins, B. Filippone, J. Jourdan, R. Mckeown, R. Milner, R. Walker, C. Woodward, P. Bosted, Z.E. Meziani, and R. Minehart, "Two-body disintegration of the Deuteron with 0.8 $1.8 \mathrm{GeV}$ Photons", WEIN 89 Symposium, Montreal.

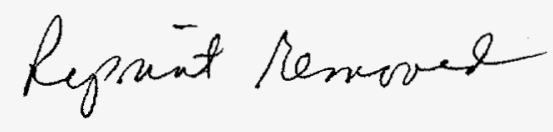




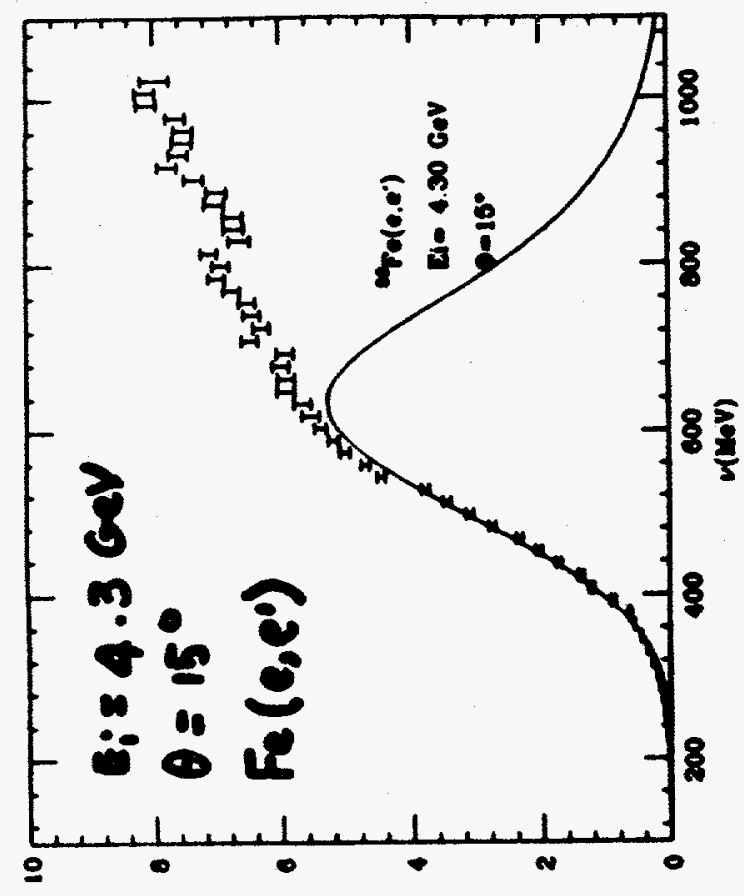

(20 neg/am) Ap Up/ep

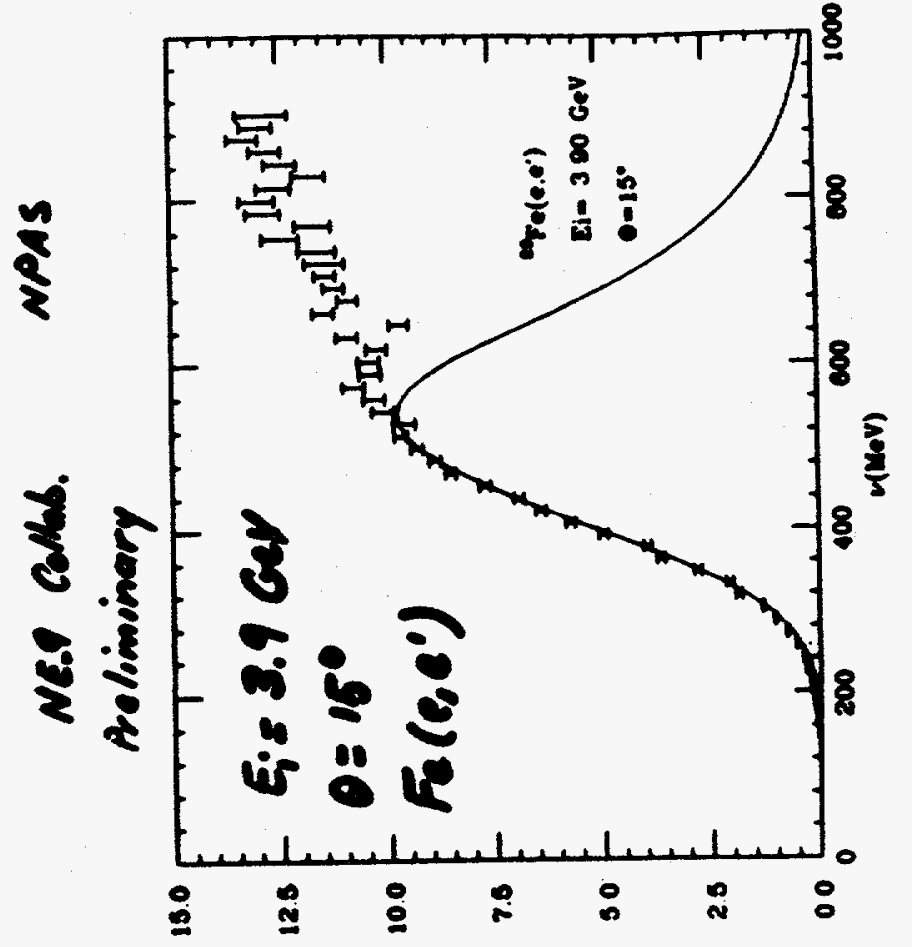

(20 Aes/AM) AP UP/OP

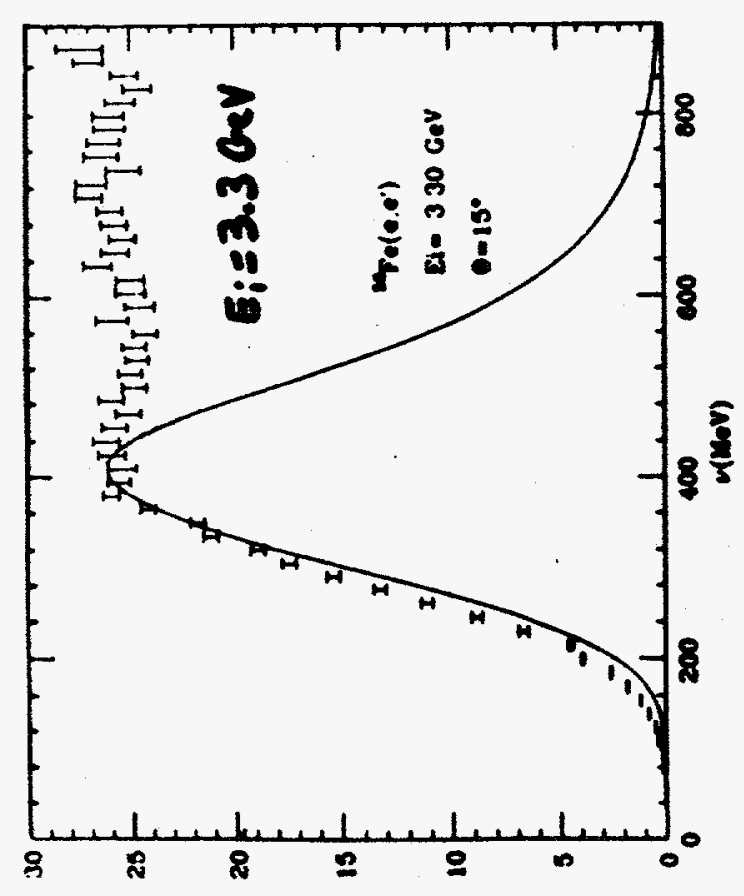

(20 sep/am n op/ap

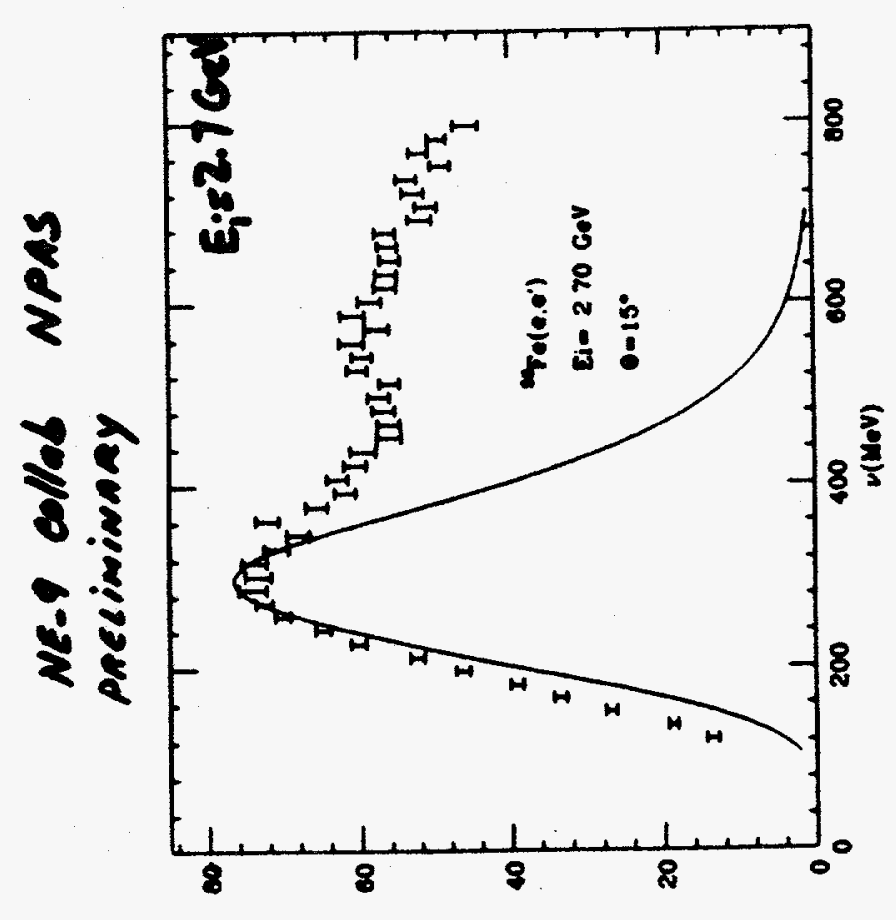

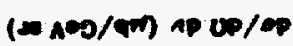

i 